\title{
Transcriptome sequencing and analysis of the entomopathogenic fungus Hirsutella sinensis isolated from Ophiocordyceps sinensis
}

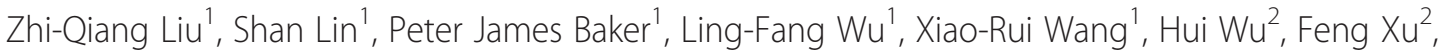 \\ Hong-Yan Wang' ${ }^{2}$, Mgavi Elombe Brathwaite ${ }^{3}$ and Yu-Guo Zheng ${ }^{1 *}$
}

\begin{abstract}
Background: Ophiocordyceps sinensis, a worm and fungus combined mixture which Hirsutella sinensis is parasitic on the caterpillar body, has been used as a traditional medicine or healthy food in China for thousands of years. $H$. sinensis is reported as the only correct anamorph of $O$. sinensis and its main active ingredients are similar to the natural O. sinensis.

Results: H. sinensis L0106, asexual strain of $O$. sinensis, was isolated and identified in this study. Three transcriptomes of $\mathrm{H}$. sinensis at different cultivation periods (growth period $3 \mathrm{~d}$, pre-stable period $6 \mathrm{~d}$ and stable period $9 \mathrm{~d}$ ) were sequenced for the first time by RNA-Seq method, and 25,511 unigenes (3d), 25,214 unigenes (6d) and 16,245 unigenes (9d) were assembled and obtained, respectively. These unigenes of the three samples were further assembled into 20,822 unigenes (All), and 62.3 percent of unigenes (All) could be annotated based on protein databases. Subsequently, the genes and enzymes involved in the biosynthesis of the active ingredients according to the sequencing and annotation results were predicted. Based on the predictions, we further investigated the interaction of different pathway networks and the corresponding enzymes. Furthermore, the differentially expressed genes (DEGs) of H. sinensis grown during different developmental stages (3d-VS-6d, 3d-VS-9d and 6d-VS-9d) were globally detected and analyzed based on the data from RNA-Seq, and 764 DEGs between 3d and 6d, 1,869 DEGs between 3d and $9 \mathrm{~d}$, and 770 DEGs between $6 \mathrm{~d}$ and $9 \mathrm{~d}$ were found, respectively.
\end{abstract}

Conclusions: This work presented here would aid in understanding and carrying out future studies on the genetic basis of $\mathrm{H}$. sinensis and contribute to the further artificial production and application of this organism. This study provided a substantial contribution and basis to further characterize the gene expression profiles of $H$. sinensis in the metabolic pathways of active ingredients.

Keywords: Ophiocordyceps sinensis, Hirsutella sinensis, Transcriptome sequencing, Metabolic pathways, Gene differential expression

\section{Background}

Ophiocordyceps sinensiss, a worm and fungus combined mixture which Hirsutella sinensis is parasitic on the caterpillar body [1], is found on the Tibetan plateau in the southwestern China [2], and widely used as one of important traditional Chinese medicines [3,4]. Modern pharmacological studies have proved that $H$. sinensis

\footnotetext{
* Correspondence: zhengyg@zjut.edu.cn

'Institute of Bioengineering, Zhejiang University of Technology, Hangzhou 310014, Zhejiang, P R China

Full list of author information is available at the end of the article
}

contains various active ingredients which have a broad therapeutic function $[5,6]$. H. sinensis attacks the caterpillar in the late autumn, and by the early summer of the following year, the caterpillar has been killed and the fruiting body of $H$. sisnensis protrudes from its head [6]. Recently, it is found that the $H$. sinensis has activities to modulate immune responses, inhibit tumour cell proliferation, enhance hepatic function, regulate insulin sensitivity and decrease plasma cholesterol levels [7-9]. As high demand for $O$. sinensis products grows and the supply of its wild type declines $[10,11]$, mycelia of $H$. sinensis grown

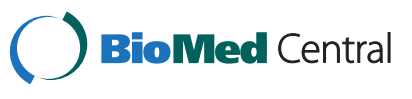


under artificial culture conditions are increasingly used in the traditional Chinese medicines. As the only correct anamorph of $O$. sinensis $[12,13], H$. sinensis could treat weakness after sickness, lung and kidney-associated diseases and sexual dysfunction [7]. It is reported that laboratory-grown $H$. sinensis mycelium has similar clinical functions and less associated toxicity compared to the wild $O$. sinensis [14]. So the fundamental research of the $H$. sinensis to investigate its function based on genetics is becoming more and more important and urgent.

Transcriptome could provide information of gene expression profiles and infer the gene functions, which has been widely applied to investigate the gene expression at RNA levels. The use of next-generation sequencing technology of transcriptome could systematically provide a complete view of expressed genes and their expression levels for the organisms at a given cultivation time, find genes and proteins involved in secondary metabolism and clarify functions of the corresponding metabolic pathways. Some methods such as serial analysis of gene expression (SAGE) [15], reassociation kinetics (Rot) [16], microarrays [17], sequencing of expressed sequence tags (ESTs) and full length transcripts [18] have been used to extensively study the transcriptomes. Gene and alternative isoform expression levels have been quantified using deep sequencing of RNAs by RNA-Seq [19,20]. RNASeq is more sensitive, both in terms of detection of lowly expressed and differentially expressed genes [21,22], and expression values from RNA-Seq correlate better with protein levels [23]. In RNA-Seq, all RNAs of a sample are randomly fragmented, reverse transcribed, ligated to adapters and then these fragments are sequenced. Gene expression levels can be estimated from the number of sequence reads deriving from each gene [24]. Expression estimates from RNA-Seq are quantitative over five orders of magnitude and replicates of mouse tissues are highly reproducible [19]. It was viable to directly analyze transcriptome of non-model organisms using RNA-Seq. Vera et al. [25] studied the transcriptome of butterflies by RNA-Seq at the condition of lacking species genetic information. RNA-Seq method can further be used to detect unknown genes, discover new transcripts, and accurately identify the variable shear loci and cSNP and UTR region [26]. Zhang et al. [27] used the paired-end RNA-Seq technology to sequence eight organs of cultivated rice, and detected 7,232 new transcription areas which have not yet been determined.

In recent years, due to the excessive excavation and high demand for $O$. sinensis, the supply of natural $O$. sinensis has been significantly reduced. Because of its important applications in the Chinese traditional medicines, it is very urgent to find new ways to meet the demand for O. sinensis. With the help of low-cost and fast sequencing technologies or approaches, the draft transcriptome sequence will facilitate understanding of the genetic basis of many traits at genome level and allow the undertaking of transcriptome-wide association studies of $H$. sinensis, which will provide theoretical foundation that $H$. sinensis is an alternative instead of the $O$. sinensis in the traditional Chinese medicines. Several features of $H$. sinensis, such as infection and lowtemperature adaption, are investigated and confirmed based on its transcriptome and accordingly experiments. The availability of transcriptome will facilitate the development of new products and more efficient production of $H$. sinensis. This work presented here would aid in understanding active ingredients metabolic pathways and carrying out the future research of this organism on the genetic level. Moreover, the fruits from this study would further contribute to the use of $H$. sinensis, protect the wild $O$. sinensis and pave the foundations for developing new drugs in biopharmacologicals.

In this study, in order to protect the endangered wild $H$. sinensis resources, save biological information and investigate the important mechanisms of this traditional Chinese medicine, three transcriptomes of $H$. sinensis under different cultivation periods were sequenced and compared for the first time. 26,577,780 reads, 27,355,558 reads and 33,619,376 reads were obtained for $3 \mathrm{~d}, 6 \mathrm{~d}$ and 9d samples, respectively. After assembling, 25,511 unigenes, 25,214 unigenes and 16,245 unigenes were obtained for $3 \mathrm{~d}, 6 \mathrm{~d}$ and $9 \mathrm{~d}$ samples, respectively. Compared with $O$. sinensis worm-part cDNA library which were clustered into 1,333 contigs and 4,172 unique sequences, as well as $O$. sinensis grass-part cDNA library which were clustered into 1,297 contigs and 3,805 unique sequences [28], $H$. sinensis has more abundant functional genes. Furthermore, we investigated and globally detected the differentially expressed genes of $H$. sinensis grown during different developmental stages based on RNA-Seq, and found 764 DEGs between $3 \mathrm{~d}$ and 6d, including 549 and 215 genes up- and down-regulated from $3 \mathrm{~d}$ to $6 \mathrm{~d}$ (FDR $\leq 0.001), 1,869$ DEGs between 3d and 9d, including 1,410 and 459 genes up- and down-regulated from $3 \mathrm{~d}$ to 9d, and 770 DEGs between 6d and 9d, including 215 and 555 genes up- and down-regulated from $6 \mathrm{~d}$ to $9 \mathrm{~d}$, respectively. Metabolic pathways of active ingredients including mannitol, cordycepin, purine nucleotides, pyrimidine nucleotides, unsaturated fatty acid, cordyceps polysaccharide and sphingolipid were further investigated based on the transcriptomes, as well as the transcriptome of genes involved in these metabolic pathways were predicted and verified according to the annotation information. The genes encoding exocellular hydrolytic enzymes such as protease, chitinase and lipase etc. which play important roles in the process of invading host, and the genes encoding low-temperature enzymes such as malate dehydrogenase, ethanol dehydrogenase and chitinase etc., 
were also predicted and cloned. The infection and cold tolerance mechanisms of $H$. sinensis were further investigated and discussed.

\section{Results}

Summary of RNA-Seq data sets

To obtain an overview of the $H$. sinensis transcriptome at different developmental stages, the RNA samples were prepared from the mycelium in different cultivation phases (3d, 6d and 9d), and poly(A)-enriched mRNA samples were subjected to high-throughput Illumina GA IIx sequencing. We totally obtained $26,577,780$ reads and 27,355,558 reads both with an average length of $90 \mathrm{nt}$ and 33,619,376 reads with an average length of $75 \mathrm{nt}$ for the samples of $3 \mathrm{~d}, 6 \mathrm{~d}$ and $9 \mathrm{~d}$, respectively. These reads were assembled with short reads assembling program SOAPdenovo, resulting in 165,480 contigs for 3d, 142,072 contigs for $6 \mathrm{~d}$ and 44,112 contigs for 9d (Table 1). The mean contig size was 178 nt (3d) with lengths ranging from $50 \mathrm{nt}$ to $6,984 \mathrm{nt}, 191 \mathrm{nt}(6 \mathrm{~d})$ with lengths ranging from $50 \mathrm{nt}$ to $6,863 \mathrm{nt}$ and $386 \mathrm{nt}$ (9d) with lengths ranging from $75 \mathrm{nt}$ to 6,037 nt. SOAPdenovo connected the contigs using $\mathrm{N}$ to represent unknown sequences between each two contigs, and then 92,721 scaffolds were made, with mean sizes of $524 \mathrm{nt}$ (3d), $520 \mathrm{nt}$ (6d) and $776 \mathrm{nt}$ (9d) (Table 1). With pariedend reads, the 35,516 scaffolds (3d) generated 25,511 unigenes (mean size: $681 \mathrm{nt}$ ), the 35,448 scaffolds (6d) generated 25,214 unigenes (mean size: $682 \mathrm{nt}$ ) and the 21,757 scaffolds (9d) generated 16,245 unigenes (mean size: $994 \mathrm{nt})$ (Table 1). Unigenes from each sample (3d, $6 \mathrm{~d}$ and $9 \mathrm{~d}$ ) were taken into further process of sequence splicing and redundancy removing with sequence clustering software to acquire non-redundant unigenes of maximal length. At last, 20,822 unigenes (All) were obtained with a mean size of 1,013 nt. To demonstrate the quality of sequencing data, we randomly selected 133 unigenes (All) and accordingly designed primers for RT-PCR amplification. In this study, all primer pairs resulted in a band of the expected size and the identity of all PCR products were confirmed by Sanger sequencing, which indicated that the transcriptome quality of $H$. sinensis was good enough for further analysis.

\section{Functional annotation}

The reads of $H$. sinensis in different cultivation periods (3d, 6d and 9d) were assembled, and then 25,511 unigenes (3d), 25,214 unigenes (6d) and 16,245 unigenes (9d) were obtained, respectively. Furthermore, these unigenes were spliced and 20,822 unigenes (All) were generated (Additional file 1: Table S1). Finally, the unigenes were carried out for COG functional annotation. For protein functional annotation, the unigene sequences were searched using BLASTx against the protein databases including non-redundant (nr), Swiss-Prot, KEGG and COG using a cut-off E-value of 1.0e-5. If the results

Table 1 Output statistics of sequencing and assembly

\begin{tabular}{llll}
\hline Samples & $\mathbf{3 d}$ & $\mathbf{6 d}$ & $\mathbf{9 d}$ \\
\hline Reads & & & All \\
Number of reads & $26,577,780$ & $27,355,558$ & $33,619,376$ \\
Read size (nt/read) & 90 & 90 & 75 \\
Total nucleotides (nt) & $2,392,000,200$ & $2,462,000,220$ & $2,521,453,200$ \\
Q20 percentage (\%) & 89.46 & 89.66 & 86.08 \\
N percentage (\%) & 0.01 & 0.00 & 0.00 \\
GC percentage (\%) & 58.19 & 57.92 & 57.00 \\
Contigs & & & \\
Number of contigs & 165,480 & 142,072 & 44,112 \\
Mean size of contigs & 178 & 191 & 386 \\
Length of all contigs (nt) & $29,423,650$ & $27,169,112$ & $17,023,492$ \\
Scaffolds & & & \\
Number of scaffolds & 35,516 & 35,448 & 21,757 \\
Mean size of scaffolds & 524 & 520 & 776 \\
Length of all scaffolds (nt) & $18,593,527$ & $18,426,448$ & $16,891,810$ \\
Unigenes & & 25,214 & 16,245 \\
Number of unigenes & 25,511 & 682 & 994 \\
Mean size of unigenes & 681 & $17,203,586$ & $16,152,293$ \\
Length of all unigenes & $17,383,818$ & & $21,085,467$ \\
\hline
\end{tabular}


of different databases conflict with each other, a priority order of nr, Swiss-Prot, KEGG and COG should be followed. Using this approach, 12,980 unigenes returned an above cut-off BLAST result (nr, 12,790 genes; SwissProt, 172 genes; KEGG, 14 genes; COG: 4 genes). Figure 1 indicates that the proportion of sequences with matches in protein databases is greater among the longer unigene sequences. Specifically, a $97.8 \%$ of match efficiency was observed for sequences longer than 2,000 bp, whereas the match efficiency decreased to about $64.8 \%$ for those ranging from 500 to $1,000 \mathrm{bp}$ and to $40.9 \%$ for sequences between 100 to 500 bp (Figure 1). The E-value distribution of the top hits in the $\mathrm{nr}$ database (12,790 unigenes) showed that $43 \%$ of the mapped sequences have strong homology (smaller than 1.0e-50), whereas $57 \%$ of the homology sequences ranged between 1.0e-5 to $1.0 \mathrm{e}-50$ (Figure 2A). For species distribution, $24 \%$ of the unigenes (hit in $\mathrm{nr}$ database) trained with sequences from the Verticillium alboatrum (Figure 2B), followed by the Penicillium chrysogenum (11\%) and Neurospora crassa (10\%).

\section{GO and COG classification}

$\mathrm{GO}$ assignments were used to classify the unigene functions of $H$. sinensis. Based on sequence homology, 1,353 unigenes were categorized into 38 functional groups (Figure 3). In terms of the biological process, unigenes involved in 'cellular process' (564 members), 'metabolic process' (600 members) and 'multi-organism process' (235 members) accounted for the majority, while unigenes involved in 'biological adhesion' ( 2 members), 'cell death' (1 member), 'growth' (4 members), 'locomotion' ( 1 member) and 'viral reproduction' (1 member) accounted for a minority. For cellular component, unigenes involved in 'cell' (526 members), 'cell part' (526 members)

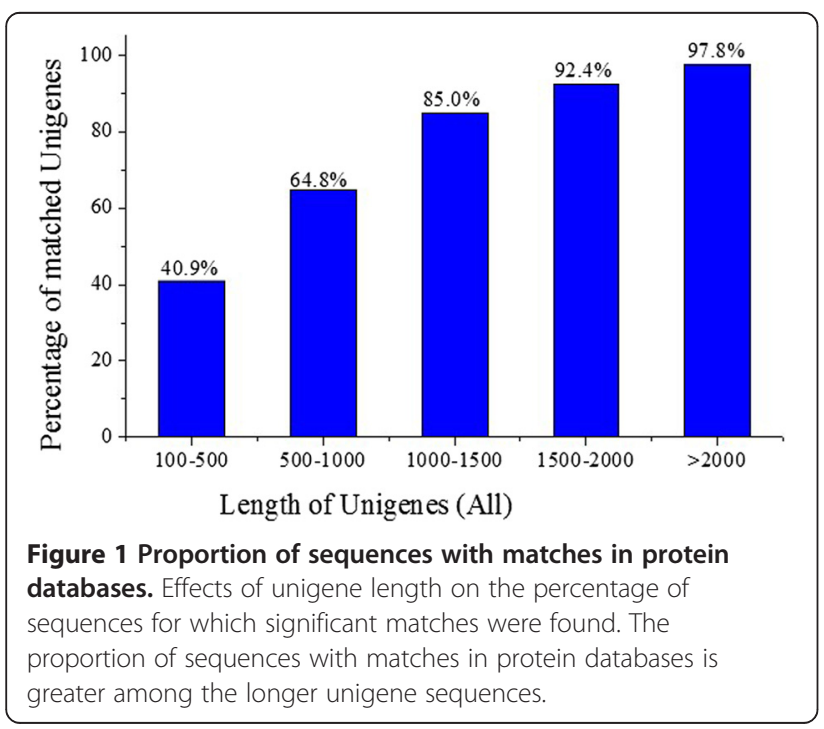

and 'organelle' (271 members) accounted for the majority, while unigenes involved in 'extra cellular region' (2 members) and 'membrane-enclosed lumen' (22 members) accounted for a minority. When the molecular function was investigated, it is found that unigenes involved in 'binding' (591 members) and 'catalytic activity' (668 members) accounted for the majority, while unigenes involved in 'antioxidant activity' ( 1 member), 'enzyme regulator activity' (7 members) and 'molecular transducer activity' (9 members) accounted for a minority (Figure 3). To further evaluate the completeness of transcriptome and the effectiveness of annotation process, the annotated sequences were screened for the genes involved in COG classifications. In total, out of $12790 \mathrm{nr}$ hits, 6,353 sequences have COG classifications (Figure 4). Among the 25 COG categories, the cluster for 'general function prediction' represents the largest group (2,273 members) followed by 'function unknown' (1,810 members) and 'transcription' (1,794 members). The following categories: Nuclear structure (4 members); Extracellular structures (12 members) and RNA processing and modification (48 members), represent the small groups. To identify the biological pathways that are active in the $H$. sinensis, we mapped the 12,980 annotated unigenes to the reference canonical pathways in KEGG, there are totally 8,724 sequences can be assigned to 159 KEGG pathways. The most representative pathways by the unigenes were 'metabolic pathways' (3,500 members), 'starch and sucrose metabolism' (1,344 members) and 'biosynthesis of secondary metabolites' (1,196 members). These annotations provide a valuable resource for investigating specific processes, functions and pathways in $H$. sinensis. COG function classification of $H$. sinensis was also compared with $O$. sinensis grass-part (OSGP) and worm-part (OSWP). The results showed that $H$. sinensi has more unigenes than both OSGP library and OSWP library in each of the COG categories indicating $H$. sinensis transcriptome has a more active expression than both OSGP and OSWP (Additional file 2: Table S2).

\section{Reference genome analysis of the $H$. sinensis transcriptome}

The comparison and analysis between reference genome and sequenced transcriptome could clarify their intrinsic link in structure, function and evolution of species, and further discover their common features and uniqueness. The transcriptome against reference genome analysis revealed an extensive expression of the whole $H$. sinensis genome (the genome of the $H$. sinensis L0106 with 102.7 $\mathrm{Mb}$ in our laboratory had sequenced, data not shown). Of the transcriptome reads, about $71.30 \%$ (3d), $70.85 \%(6 \mathrm{~d})$ and $78.21 \%(9 \mathrm{~d})$ was mapped to the reference genome of $H$. sinensis. Moreover, $33.00 \%$ (3d), $31.55 \%(6 \mathrm{~d})$ and $32.92 \%(9 \mathrm{~d})$ could be mapped to reference genes (Additional file 3: Table S3). There were 


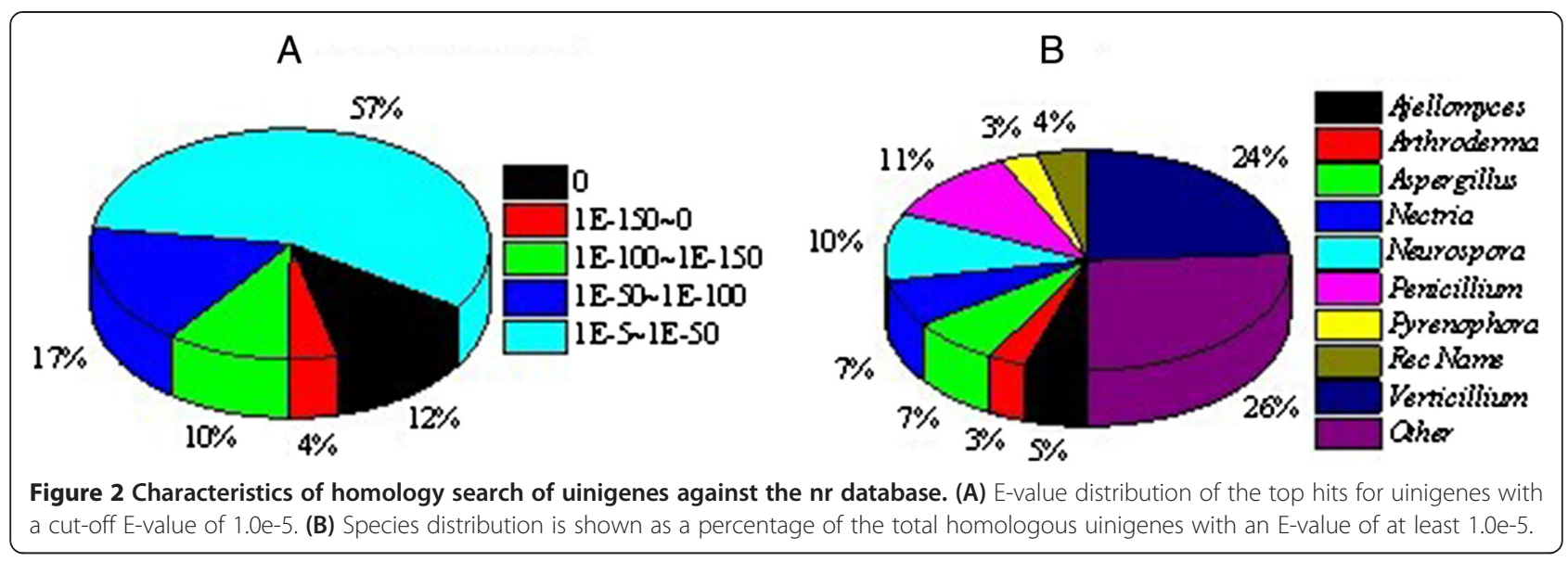

13,031,334 perfect matched reads and accounted for 49.03\% for $3 \mathrm{~d}$ samples, $19,380,240$ perfect matched reads and accounted for $70.85 \%$ for $6 \mathrm{~d}$ samples, and $10,185,645$ perfect matched reads and accounted for $60.59 \%$ for $9 \mathrm{~d}$ samples, respectively. The mapped reads were classified by both of mismatch number and uniqueness of alignment position. There were 17,285,218 unique matched reads and 1,664,653 multi-position matched reads for $3 \mathrm{~d}$ samples, $17,445,855$ unique matched reads and 1,934,385 multi-position matched reads for $6 \mathrm{~d}$ samples, 12,094,781 unique matched reads and 1,052,137 multi-position matched reads for $9 \mathrm{~d}$ samples, respectively. In order to assess the randomness of RNA-Seq, the randomness of mRNA fragmentation was evaluated with the reads distribution in reference genes. The total number of reads aligned to reference genes was counted, and the reads were located in relative position in reference gene (Figure 5). More than $80 \%$ reads of $3 \mathrm{~d}, 6 \mathrm{~d}$ and $9 \mathrm{~d}$ were located in relative position from 0.2 to 0.9 , the reads number of $3 \mathrm{~d}$ in this region distributed random and was around $1.0 \mathrm{e}+05$, the reads number of $6 \mathrm{~d}$ with the relative position at 0.2 was around $0.5 \mathrm{e}+05$ but the relative position at 0.9 was around $1.5 \mathrm{e}+05$, and the reads number of $9 \mathrm{~d}$ with the relative position at 0.2 was around $0.3 \mathrm{e}+05$ but the relative position at 0.9 was around $1.3 \mathrm{e}+05$, which indicated that the distribution quality of reads of $3 \mathrm{~d}$ was better than both $6 \mathrm{~d}$ and 9d. Therefore, the distribution of reads in the reference genes was homogeneous and the randomness of fragmentation was good, it indicated that fragmentation of mRNA was performed well and

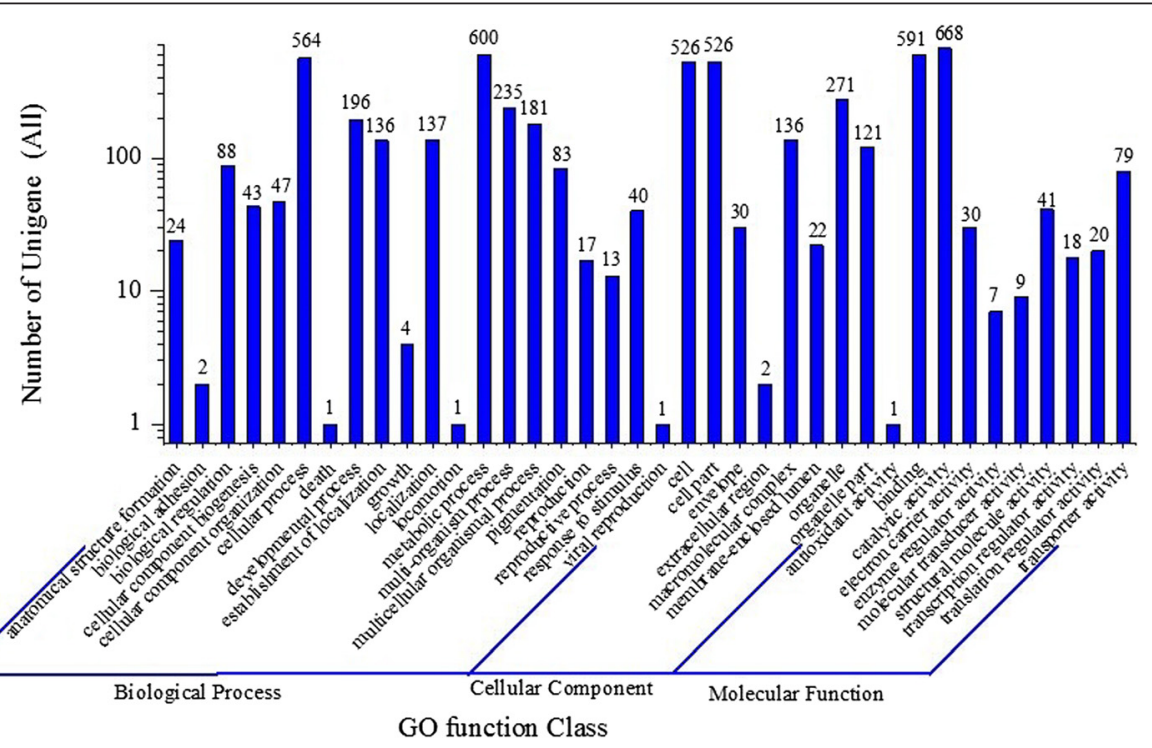

Figure 3 Histogram presentation of Gene Ontology classification. GO has three ontologies: molecular function, cellular component and biological process. It indicates the number of unigenes in a category. The basic unit of GO is GO-term, and every GO-term belongs to a type of ontology. GO functional analysis provided GO functional classification annotation for DEGs as well as GO functional enrichment analysis for DEGs. 


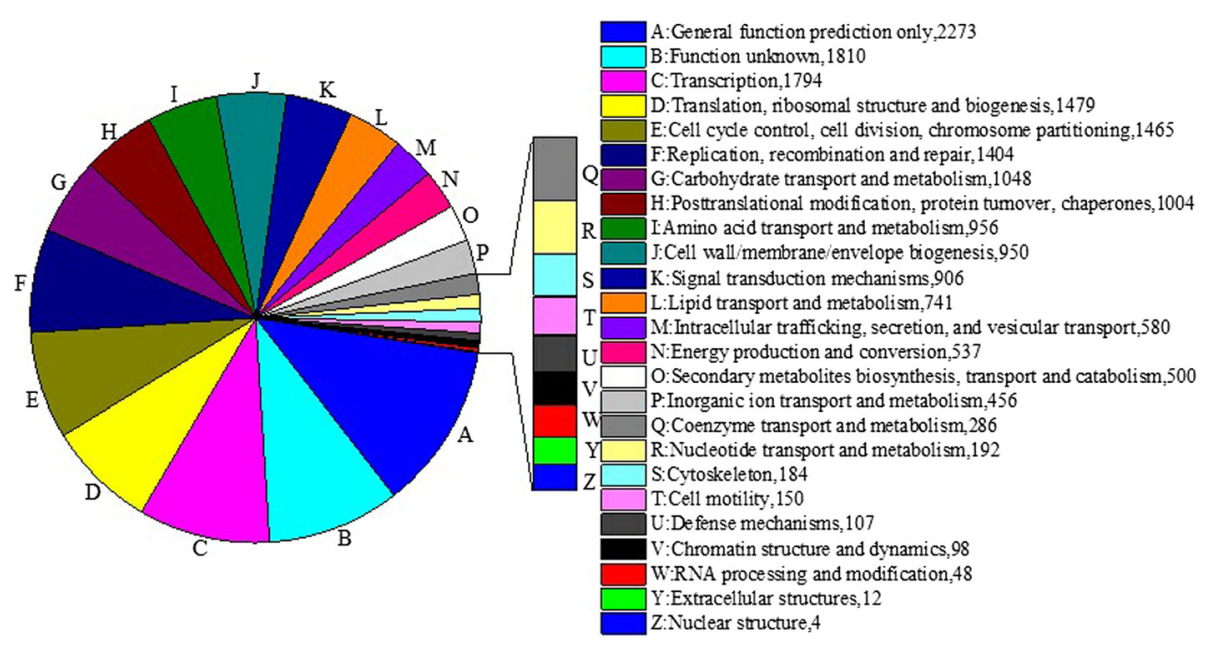

Figure 4 Cluster of orthologous groups (COG) function classification of unigene sequence.

conducive to transcriptome analysis. Gene coverage is the percentage of a gene covered by reads, this value equals to ratio of the number of bases in a gene covered by unique mapping reads to number of total bases in the gene. We found that 7,496 genes were covered by the reads of $3 d$, $6 \mathrm{~d}$ and $9 \mathrm{~d}$ transcriptomes, and the genes with more than $70 \%$ of the gene coverage were over $90 \%$. Number of RPKM (defined in Method) was calculated to quantify the overall transcriptional activity of the genes (Figure 6). The results showed that RNA-Seq data obtained in this study was more sensitive, which displayed a comprehensive landscape of the $H$. sinensis transcriptome.

\section{Prediction and optimization of novel transcripts}

Novel transcripts can be found by high throughput sequencing since present databases may be incomplete. Gene models with a length more than 150 bp found in intergenic regions (200 bp away from upstream or downstream genes) were thought to be candidates of the novel transcripts. Extensive reads mapping and clustering revealed that 2,867 (3d), 2,738 (6d) and 2,744 (9d) novel transcripts with the significant expression levels were above the surrounding intergenic region, of which $40.84 \%$ (3d), 41.78\% (6d) and 39.18\% (9d) were longer than $500 \mathrm{bp}$ and provided a sufficient candidate number of novel transcripts (Figure 7), and most of these novel transcripts belong to the non-coding RNA. We also globally mapped the $5^{\prime}$ - and $3^{\prime}$-boundaries of $H$. sinensis genes by searching for a sharp reduction of RNA-Seq reads signals at both ends of annotated genes. Genes whose $5^{\prime}$ - or 3'-boundaries overlap with other genes were excluded from the analysis. The results defined or extended 5' - or (and) 3'-boundary regions for 5,052 transcribed genes in $H$. sinensis transcriptome of $3 \mathrm{~d}$, 5,109 transcribed genes in $H$. sinensis transcriptome of $6 \mathrm{~d}$, and 5,061 transcribed genes in $H$. sinensis transcriptome of $9 \mathrm{~d}$ (Figure 8).

\section{Alternative splicing in $H$. sinensis}

Alternative splicing (AS) is a mechanism brings remarkable diversity to proteins which make it possible for a gene to generate different mRNA transcripts and then

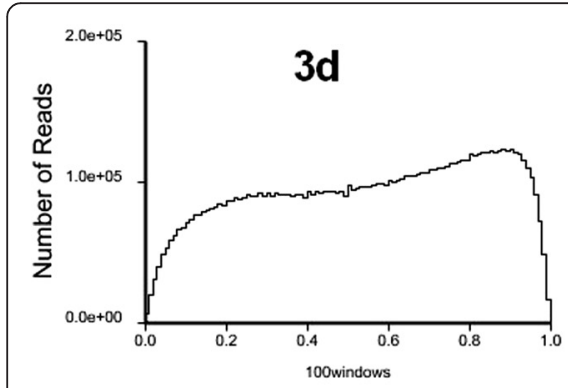

Relative Position in Gene( $\left.5^{\prime}->3^{\prime}\right)$

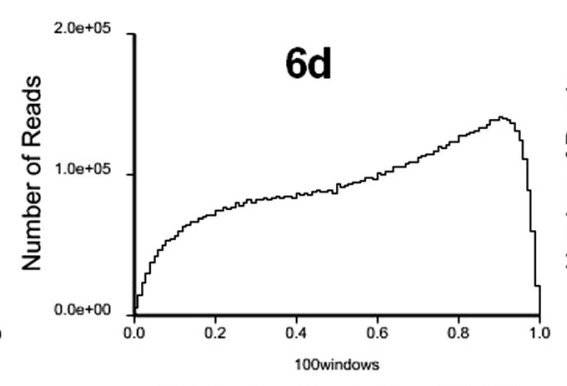

Relative Position in Gene(5'->3')

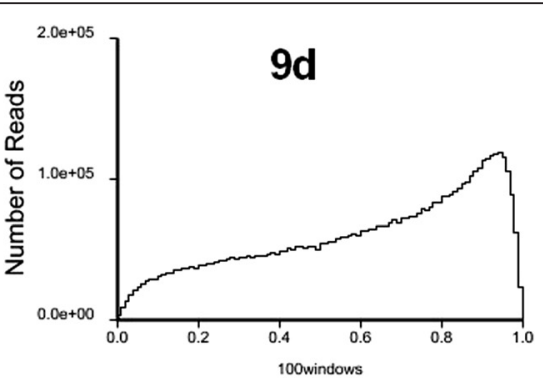

Relative Position in Gene( $\left.5^{\prime}->3^{\prime}\right)$

Figure 5 Distribution statistics of $\boldsymbol{H}$. sinensis transcriptome reads mapped to reference gene. The randomness of $3 \mathrm{~d}$ transcriptome is the best, followed with $6 \mathrm{~d}$ and $9 \mathrm{~d}$. 


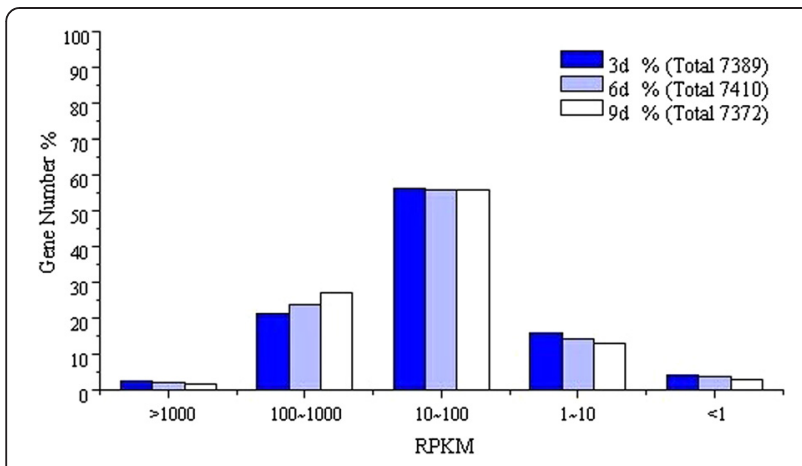

Figure 6 Distribution statistics of $H$. sinensis transcriptome genes expression levels. translate into distinguishable proteins [29-31]. Though alternative splicing is known to be universal in eukaryotes, we may underestimate the number of genes that undergo alternative splicing. Recently, many new alternative splicing events were discovered in human [20,32,33], mouse [19,34] and Arabidopsis [35]. Alternative splicing events mostly occur on the genes which involved in signal transduction and expression regulation, mechanisms of cell differentiation and apoptosis could be clarified by studying these alternative splicing events. To assess the genome-wide extent of AS events in H. sinensis, with different developmental stages (3d, $6 \mathrm{~d}$ and 9d), we performed computational analysis to determine the known and putative splicing junctions and then to identify sequence reads mapping to these regions using stringent criteria. 5,203 genes of $H$. sinensis underwent AS with 6,324 AS events (Figure 9), 1,798 genes of 3d samples underwent AS with 2,213 AS events, 1,813 genes of 6d samples underwent AS with 2,212 AS events, and 1,590 genes of 9d samples underwent AS with 1,899 AS events, respectively in four common types of AS events, including Exon skipping (ES), Intron retention (IR), Alternative $5^{\prime}$ splice site (A5SS) and

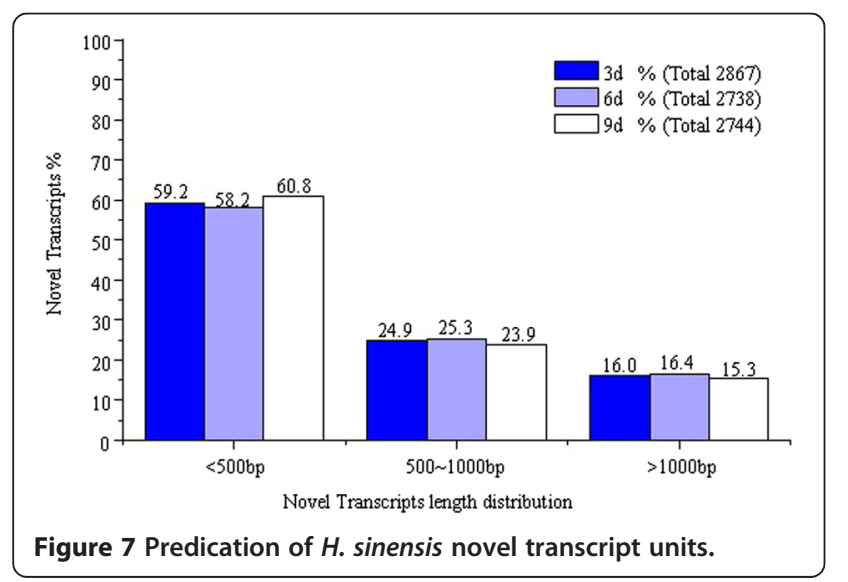

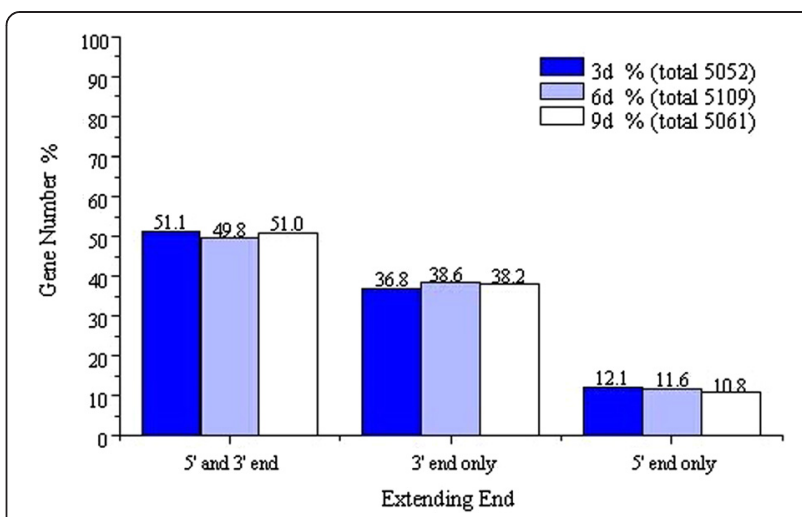

Figure 8 Refinement of $H$. sinensis transcript gene structures.

Alternative $3^{\prime}$ splice site (A3SS). For the data obtained in this study, $24.33 \%$ (3d), $24.49 \%$ (6d) and $21.57 \%$ (9d) of $H$. sinensis genes were estimated to undergo AS.

In this study, Ustilago maydis [36] and Magnaporthe grisea [37], two important fungal pathogens to plant, shared similar infection process with $H$. sinensis, are chosen as references to develop transcriptome analysis. The IR in $H$. sinensis is the predominant form of AS, accounting for $59.29 \%$ (3d), $62.87 \%$ (6d) and $65.41 \%$ (9d) of all AS isoforms, indicating $H$. sinensis has a richer gene activity compared with $U$. maydis and $M$. grisea.

\section{Gene expression difference analysis}

Studies on the up- and down-regulated DEGs of $H$. sinensis were carried out by GO annotation, the results were shown in Table 2. The number of up- and down-regulated DEGs were further compared among $3 \mathrm{~d}-\mathrm{VS}-6 \mathrm{~d}, 9 \mathrm{~d}-\mathrm{VS}-3 \mathrm{~d}$ and $9 \mathrm{~d}-\mathrm{VS}-6 \mathrm{~d}$ by GO component ontology, GO function ontology and GO process ontology and the details were shown in Table 3, Table 4 and Table 5, respectively. There were 467 DEGs and 255 upregulated genes in GO component ontology, 1,040 DEGs and 423 up-regulated genes in GO function ontology,

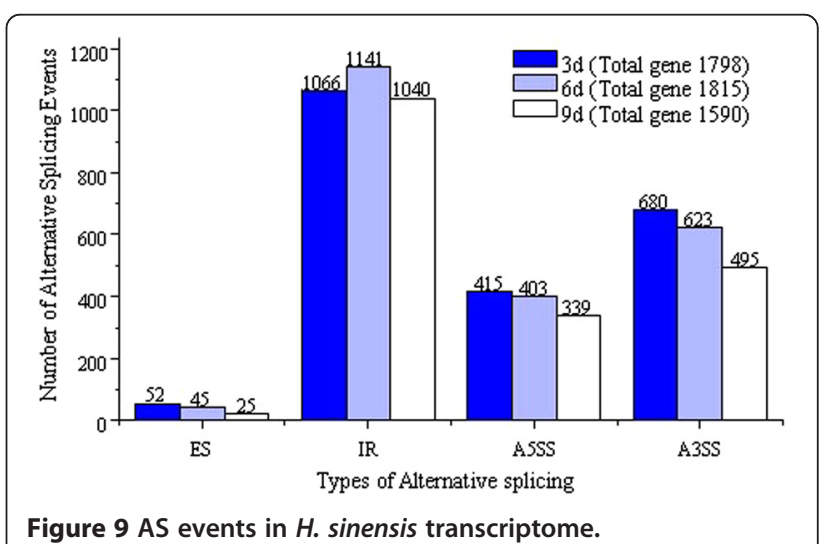


Table 2 Statistic chart of $\boldsymbol{H}$. sinensis DEGs carried out by GO annotation

\begin{tabular}{lllll}
\hline $\begin{array}{l}\text { GO } \\
\text { annotation }\end{array}$ & $\begin{array}{l}\text { Up-regulated } \\
\text { DEGs }\end{array}$ & $\begin{array}{l}\text { Down-regulated } \\
\text { DEGs }\end{array}$ & $\begin{array}{l}\text { Component } \\
\text { ontology }\end{array}$ & $\begin{array}{l}\text { Function } \\
\text { ontology }\end{array}$ \\
\hline 3d-VS-6d & 203 & 91 & 117 & 259 \\
9d-VS-3d & 249 & 435 & 279 & 557 \\
9d-VS-6d & 117 & 158 & 96 & 235 \\
\hline
\end{tabular}

and 568 DEGs and 240 up-regulated genes in GO process ontology. A large number of differentially expressed genes were up-regulated in GO function ontology, especially in terms of 'catalytic activity', 'oxidoreductase activity' and 'hydrolase activity', which indicated $H$. sinensis could secrete large amounts of enzymes involved in secondary metabolism pathway, infection mechanism and cold tolerance mechanism. 36 down-regulated genes (3d-VS-6d), 63 up-regulated genes (9d-VS-3d), and 13 up-regulated genes (9d-VS-6d) were involved in ribonucleoprotein complex by GO component ontology, indicating that the speed and efficiency of protein synthesis in stable period were higher than growth period. 36 down-regulated genes (3d-VS-6d), 63 up-regulated genes (9d-VS-3d), and 12 up-regulated genes (9d-VS-6d) were involved in structural molecule activity by GO function ontology, showing that structural molecule in stable period was more stable than growth period. 35 downregulated genes (3d-VS-6d), 58 down-regulated genes (9d-VS-3d), and 27 down-regulated genes (9d-VS-6d) were involved in metabolic process by GO process ontology, which demonstrated that genes involved in metabolic processes were more abundant in stable period. Subsequently, ExtendGene, Exon skipping and Intron retention analysis of $3 \mathrm{~d}, 6 \mathrm{~d}$ and $9 \mathrm{~d}$ were absolutely compared, respectively. There were common 4,177 ExtendGenes, 10 skipping Exons and 460 retentional Introns among 3d, 6d and 9d. Comparisons among of

Table 3 Statistics chart of up- and down-regulated $H$. sinensis DEGs by GO component ontology

\begin{tabular}{|c|c|c|c|c|c|c|}
\hline \multirow[t]{2}{*}{ GO component ontology } & \multicolumn{2}{|c|}{$3 d-V S-6 d$} & \multicolumn{2}{|c|}{$9 d-V S-3 d$} & \multicolumn{2}{|c|}{$9 d-V S-6 d$} \\
\hline & Up & Down & Up & Down & Up & Down \\
\hline Ribonucleoprotein complex & 0 & 36 & 63 & 1 & 13 & 0 \\
\hline Intrinsic to membrane & 23 & 5 & 10 & 32 & 6 & 10 \\
\hline $\begin{array}{l}\text { Intracellular membrane-bounded } \\
\text { organelle }\end{array}$ & 11 & 4 & 25 & 22 & 9 & 8 \\
\hline Membrane & 7 & 2 & 4 & 16 & 3 & 7 \\
\hline Cell part & 8 & 0 & 7 & 22 & 2 & 9 \\
\hline Intracellular part & 5 & 3 & 24 & 13 & 9 & 3 \\
\hline Organelle inner membrane & 3 & 2 & 5 & 5 & 2 & 1 \\
\hline Intracellular & 2 & 1 & 1 & 4 & 0 & 1 \\
\hline Protein complex & 1 & 2 & 8 & 2 & 4 & 1 \\
\hline
\end{tabular}

Table 4 Statistics chart of up- and down-regulated $H$. sinensis DEGs by GO function ontology

\begin{tabular}{|c|c|c|c|c|c|c|}
\hline \multirow[t]{2}{*}{ GO function ontology } & \multicolumn{2}{|c|}{$3 d-V S-6 d$} & \multicolumn{2}{|c|}{$9 d-V S-3 d$} & \multicolumn{2}{|c|}{$9 d-V S-6 d$} \\
\hline & Up & Down & Up & Down & Up & Down \\
\hline Structural molecule activity & 0 & 36 & 63 & 0 & 12 & 0 \\
\hline Catalytic activity & 22 & 5 & 10 & 55 & 7 & 24 \\
\hline Oxidoreductase activity & 19 & 6 & 17 & 27 & 15 & 13 \\
\hline Hydrolase activity & 20 & 4 & 4 & 24 & 6 & 6 \\
\hline Binding & 16 & 6 & 11 & 44 & 5 & 17 \\
\hline Transition metal ion binding & 17 & 0 & 7 & 29 & 2 & 8 \\
\hline Metal ion binding & 11 & 3 & 10 & 20 & 4 & 9 \\
\hline Transferase activity & 12 & 2 & 10 & 31 & 7 & 12 \\
\hline Iron ion binding & 12 & 1 & 1 & 14 & 3 & 6 \\
\hline Nucleic acid binding & 8 & 4 & 22 & 20 & 5 & 4 \\
\hline Coenzyme binding & 7 & 2 & 3 & 9 & 1 & 2 \\
\hline Cation binding & 3 & 5 & 10 & 6 & 4 & 2 \\
\hline Nucleoside-triphosphatase activity & 6 & 2 & 5 & 14 & 4 & 4 \\
\hline Lyase activity & 3 & 2 & 1 & 3 & 1 & 2 \\
\hline Kinase activity & 4 & 1 & 9 & 4 & 4 & 2 \\
\hline Structural molecule activity & 0 & 36 & 63 & 0 & 12 & 0 \\
\hline
\end{tabular}

alternative $5^{\prime}$ splice site, alternative 3 ' splice site and the number of transcripts analysis of $3 \mathrm{~d}, 6 \mathrm{~d}$ and $9 \mathrm{~d}$ showed that there were common 131 alternative $5^{\prime}$ splice sites, 173 alternative $3^{\prime}$ splice sites and 7,239 transcripts among 3d, 6d and 9d. Finally, the comparison of differential expression genes, up- and down-regulated genes analysis of 3d-VS-6d, 9d-VS-3d and 9d-VS-6d were carried

Table 5 Statistics chart of up- and down-regulated H. sinensis DEGs by GO process ontology

\begin{tabular}{|c|c|c|c|c|c|c|}
\hline \multirow[t]{2}{*}{ GO Process ontology } & \multicolumn{2}{|c|}{$3 d-V S-6 d$} & \multicolumn{2}{|c|}{$9 d-V S-3 d$} & \multicolumn{2}{|c|}{$9 d-V S-6 d$} \\
\hline & $\overline{U p}$ & $\overline{\text { Down }}$ & $\overline{U p}$ & $\overline{\text { Down }}$ & $\overline{U p}$ & Down \\
\hline Metabolic process & 6 & 35 & 8 & 58 & 10 & 27 \\
\hline Gene expression & 0 & 34 & 64 & 2 & 14 & 2 \\
\hline Transport & 19 & 3 & 9 & 31 & 7 & 13 \\
\hline Transcription & 14 & 1 & 2 & 25 & 0 & 9 \\
\hline Establishment of localization & 6 & 1 & 6 & 8 & 3 & 1 \\
\hline Carboxylic acid metabolic process & 6 & 0 & 0 & 11 & 0 & 5 \\
\hline Protein modification process & 2 & 1 & 3 & 12 & 2 & 1 \\
\hline Carboxylic acid metabolic process & 6 & 0 & 0 & 11 & 0 & 5 \\
\hline Translation & 0 & 3 & 8 & 3 & 0 & 2 \\
\hline Response to stimulus & 1 & 3 & 9 & 1 & 3 & 0 \\
\hline Cellular metabolic process & 2 & 1 & 3 & 4 & 2 & 1 \\
\hline Glucose catabolic process & 0 & 0 & 8 & 0 & 5 & 0 \\
\hline Primary metabolic process & 0 & 0 & 2 & 6 & 3 & 3 \\
\hline DNA metabolic process & 0 & 0 & 5 & 2 & 2 & 3 \\
\hline
\end{tabular}


out. There were common 113 differential expression genes, while no common up- and down-regulated genes among 3d-VS-6d, 9d-VS-3d and 9d-VS-6d (Additional file 4: Figure S1).

\section{Functional analysis of differential gene expression based on RNA-Seq data}

So far, the molecular mechanisms resulting in various kinds of functional complexity in $H$. sinensis mycelium have not been illuminated [38,39]. To understand this molecular mechanisms, the DEGs of $H$. sinensis grown during different developmental stages (3d-VS-6d, 3d-VS$9 \mathrm{~d}$ and $6 \mathrm{~d}-\mathrm{VS}-9 \mathrm{~d}$ ) were also used to investigate the gene functions based on the differential gene expression level in different culture times. We found 764 DEGs between $3 \mathrm{~d}$ and $6 \mathrm{~d}$, including 549 and 215 genes up- and downregulated from $3 \mathrm{~d}$ to $6 \mathrm{~d}$ (FDR $\leq 0.001$ ), 1,869 DEGs between $3 \mathrm{~d}$ and $9 \mathrm{~d}$, including 1,410 and 459 genes up- and down-regulated from 3d to 9d, and 770 DEGs between $6 \mathrm{~d}$ and 9d, including 215 and 555 genes up- and downregulated from $6 \mathrm{~d}$ to $9 \mathrm{~d}$, respectively (Figure 10). GO functional enrichment analysis revealed that genes upregulated during the developmental stages of $H$. sinensis were mainly involved in 'structural molecule activity', 'ribonucleoprotein complex', 'macromolecular complex', 'gene expression' and 'intrinsic to membrane' (Corrected $p$-value $\leq 0.05$ ) (Additional file 4: Figure S2), suggesting these up-regulated genes played important roles and promoted the formation of cell structures in the process of mycelia reproduction. In addition, the KEGG metabolic pathway analysis indicated that the genes upregulated from $3 \mathrm{~d}$ to $6 \mathrm{~d}$ were specifically located in the pathways of 'ribosome', 'caprolactam degradation', 'metabolic pathways', 'nitrogen metabolism' and 'fatty acid metabolism', while genes up-regulated from $6 \mathrm{~d}$ to 9d were mainly associated with 'ribosome,' 'metabolic pathways', 'phenylalanine, tyrosine and tryptophan biosynthesis' and 'biosynthesis of secondary metabolites'

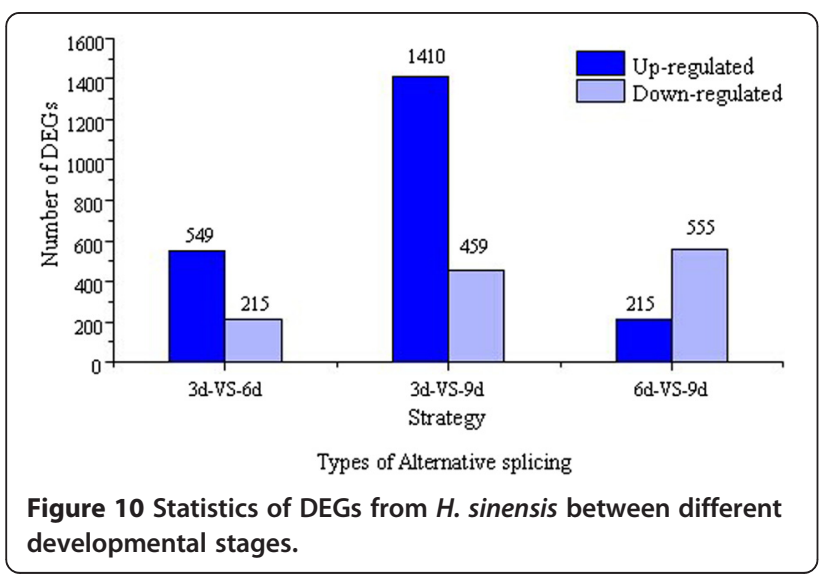

(Additional file 4: Figure S3). The results indicated that these up-regulated genes in such life stage mainly promoted secondary metabolism and biosynthesis of active ingredients, which was consistent with the description of the $H$. sinensis life cycle (Additional file 4: Figure S4). Therefore, these analyses indicated that $H$. sinensis drastically altered the manner of gene expression during the developmental stages to produce numerous functional components.

\section{Secondary metabolism analysis and verification}

According to the metabolic pathways from annotated KEGG analysis based on the transcriptome, 7 main metabolic pathways of active ingredients including mannitol, cordycepin, purine nucleotides, pyrimidine nucleotides, unsaturated fatty acid, cordyceps polysaccharide and sphingolipid in $H$. sinensis were predicted, and the genes involved in these pathways were further cloned and expressed to verify the predictions. Using the glycolytic pathway (map00010) and fructose-mannose pathway (map00051) as references, mannitol metabolic pathway of $H$. sinensis was predicted (Additional file 4: Figure S5). However, the mannitol-1-phosphatase converting mannitol-1-P into mannitol is not found, which indicates that other unknown phosphatases take place the role of mannitol-1-phosphate to generate mannitol. Subsequently, 6 hexokinase genes, 3 glucose phosphate isomerise genes and 1 mannitol-1-P dehydrogenase gene involved in mannitol metabolic pathway were successfully cloned (Additional file 4: Figure S6), and corresponding proteins which expressed in E. coli BL21 were detected by Sodium Dodecyl Sulfate Polyacrylamide Gel Electrophoresis (SDS-PAGE) (Additional file 4: Figure S7). In the KEGG, the biosynthesis of adenosine (map00230) has been confirmed. To date, there were still no literatures about the biosynthesis of cordycepin from adenosine [40]. It is found that in the pathway of cordycepin of $H$. sinensis there is special simple sugars which was called cordycepose, we speculated that cordycepose and adenosine exchanged the glycosylation by the function of N-glycosylation lyase, and then generated cordycepin (Additional file 4: Figure S8). The genes involved in cordycepin metabolic pathway were further verified by PCR, 1 ADP-ribose pyrophosphatase gene, 2 ribosephosphate pyrophosphokinase genes, $25^{\prime}$-nucleotidase genes and $1 \mathrm{~N}$-glycosylase gene etc. were successfully cloned (Additional file 4: Figure S9), and corresponding proteins which expressed in E. coli BL21 were also detected by SDS-PAGE (Additional file 4: Figure S10). Furthermore, purine biosynthesis pathway of $H$. sinensis was predicted according to the previously reported purine metabolic pathway (map00230). It is found that purine metabolic pathway starts from adenosine and ends with urate after 7 steps of catalysis (Additional file 4: 
Figure S11), and genes which encoding purine nucleosidase and adenosine kinase etc. were cloned (Additional file 4: Figure S12), and then the corresponding proteins were successfully expressed (Additional file 4: Figure S13). For the pyrimidine metabolism pathway (map00240) based on KEGG, the biosynthesis pathway which starts from L-glutamine and ends with uridylic acid, cytidylic acid, thymidylic acid etc. in $H$. sinensis was analyzed and verified (Additional file 4: Figure S14), uridine triphosphate, cytidine triphosphate and cytidine diphosphate were intermediate products in this pathway. Then the biosynthesis pathways which starts from acetyl-CoA and hexadecanoate and ends with docosahexaenoic acid and butyryl-[acp] were obtained in the summary of the KEGG fatty acid biosynthesis pathway (map00061) and unsaturated fatty acids biosynthesis pathway (map01040) (Additional file 4: Figure S15), hexadecanoate was generated by fatty acid biosynthesis pathway and then used as a starting material to promote the unsaturated fatty acids biosynthesis. Moreover, based on the fructose and mannose metabolism pathway (map00051), galactose metabolism pathway (map00052), N-glycan biosynthesis pathway (map00510) and sphingolipid metabolism pathway (map00600) from the KEGG, the biosynthesis pathway of cordyceps polysaccharide which starts from Dglucose and D-galactose and ends with UDP-glucose and (GlacNAc)2(Man)5(Asn)1 (Additional file 4: Figure S16), as well as the sphingolipid biosynthesis pathway which starts from palmitoyl CoA andends with sphingosine (Additional file 4: Figure S17), could be predicted and verified in the same manner as described above in this study.

\section{Discussion}

The use of $O$. sinensis has a long history both in traditional Chinese medicine and Tibetan medicine in China. The huge market demand has led to over harvesting and severe devastation of fragile alpine environments, however, O. sinensis grows slowly on artificial media and attempts at solid cultivating the fungus to produce fruiting bodies have consistently failed [40]. To meet the requirement of $O$. sinensis from the market, mycelia of $H$. sinensis grown under artificial liquid cultivation are increasingly used as an alternative in the medicinal products. As the anamorph of $O$. sinensis [12,13], $H$. sinensis has activities to modulate immune responses, inhibit tumour cell proliferation, enhance hepatic function, regulate insulin sensitivity and decrease plasma cholesterol levels, this makes it become more and more valuable in pharmacology. Studies have demonstrated the diverse bioactive ingredients and broad medical effects of $H$. sinensis, but there were little reports which demonstrated genetic information of $H$. sinensis. Therefore, the fundamental research of the $H$. sinensis to investigate and preserve its genetic information based on RNA-Seq is becoming more and more important and urgent.

Next generation sequencing has opened the door to transcriptome analysis of non-model organisms [41]. The growing number of species for which significant genetic resources are available is sparking a new era of study in which fundamental genetic questions underlying phenotypic evolution, adaptation and speciation can be addressed [41]. In this study, the next-generation sequencing technology was applied to $H$. sinensis transcriptome sequencing, and the unigenes generated from transcriptomes under different culture periods were systematically investigated. To our knowledge, this is first de novo assembly of the $H$. sinensis transcriptome from a short read sequencing platform and the results are presented that allows high throughput analysis and further research for comparative transcriptomics. Being an insect pathogen, $H$. sinensis contains thousands of genes putatively involved in interactions with insect hosts. Assembled unigenes were firstly aligned by blastx to protein databases like nr, Swiss-Prot, KEGG and COG, and the information about putative heterozygous nucleotide variations offered intriguing leads for the analysis of transcriptomic events and their effects on biological functions of $H$. sinensis. This study gave a new way to find secondary metabolism genes and clarify the functions of the corresponding metabolic pathways in $H$. sinensis. In addition, this study also provided a scientific basis for medicinal mechanism of $H$. sinenesis and effective protection for the sustainable use of $O$. sinensis resources. The availability of transcriptome sequence opens new avenues for new exploration, application and improvements of $H$. sinensis. It will lead to the identification and manipulation of candidate genes or genomic regions to generate the new ways to synthesize new compounds with potentials in pharmaceuticals, and pave foundation for development of new drugs for the pharmaceutical manufacturing or provide the theoretical basis for the sustainable use of $O$. sinensis.

The sequences of the $H$. sinensis transcriptome were captured by preparing RNA-Seq libraries from three different cultivation periods. Compared with worm-part and grass-part of $O$. sinensis cDNA libraries, $H$. sinensis transcriprome contained more genes which involved in translation, transcription and cell division etc. [28]. This study indicated that $H$. sinensis is more active in translation, transcription and cell division etc. Compared to other insect pathogens which have similar infection processes and life cycles to $H$. sinensis, such as $P$. chrysogenum [42], Aspergillus niger [43], Metarhizium anisopliae [44], M. acridum [44], C. militaris [40] and asexual strain of $O$. sinensis [39], $H$. sinensis has similar quantities of functional genes involved in transportation, cell 
metabolism, transcription and protein fate, while has larger quantities of functional genes involved in cell component, homeostasis and protein synthesis. This phenomenon indicates that $H$. sinensis could secret and transport more proteins for infecting the host larvae and propagating in host vivo as compared to these fungi.

The life cycle of $H$. sinensis is poorly understood aside from knowing that $H$. sinensis infects $H$. armoricanus pupae [45]. Its survival in soil may depend on the asexual stage of $O$. sinensis which provides resilient longlived ascospores as described in other fungi [46]. As the asexual type of $O$. sinensis, in the nature, $H$. sinensis mainly infects host larvae in the soil through the skin of the head by the effects of enzymes and mechanical force when $H$. sinensis grows $[47,48]$. It is reported that the infection process of $H$. sinensis can be divided into three stages including attaching to the epidermis of larvae, penetrating body wall and propagation in vivo causing the death of host. The entire process includes host recognition, mechanical damage, nutrient competition, disturbing metabolism, secretion of toxins and the damaging the tissue structure of the host, these multi-factors interaction ultimately lead to host death and form the matured O. sinensis [49]. In the process of invading the body wall of insect, $H$. sinensis secretes many kinds of exocellular hydrolytic enzymes such as protease [50], chitinase [51] and lipase etc. which play important roles in the process of invading. Degradation of proteases is not only helpful to the mycelium penetration, but also provides nutrients for mycelial growth [52]. After screening transcriptome of $\mathrm{H}$. sinensis, 157 proteases, 52 chitinases and 124 lipases were predicted and confirmed, comparing to $O$. sinensis whose gene families encoding 19 cuticle degrading proteases and 9 chitinases [39], indicating that $H$. sinensis has stronger infecting virulence. 7 chitinase genes, 7 protease genes and 3 lipase genes were successfully cloned and expressed in E. coli BL21 and the corresponding enzyme activities were successfully detected. Therefore, the results obtained in this study demonstrated that $H$. sinensis could produce these enzymes to accomplish the invasion.

Studies based on the molecular level have confirmed that nucleosides [53], sterols [54], polysaccharides [55] and mannitol [54] were the material basis for pharmacological effects of $H$. sinensis. At present, there are few reports about metabolic pathways both in $H$. sinensis and $O$. sinensis, so it is much more necessary and urgent to investigate metabolic pathways which will provide new information and method to further regulate, control and optimize the fermentation process to obtain $H$. sinensis mycelium with high quality. In order to model the biosynthesis of cordycepin, Zheng et al. [40] constructed the purine metabolic pathway in C. militaris based on the KEGG annotations, and suggested that the biosynthesis of cordycepin proceeds through a reductive mechanism as described for the formation of 2 '-deoxyadenosine [56]. Hu et al. [46] found O. sinensis has multiple polyketide synthases, modular non-ribosomal peptide synthases and terpene cyclases for producing an array of secondary metabolites, these enzymes likely play roles after the latent period when the fungus is colonizing and killing the host, and they are also potential candidates for production of pharmacologically active ingredients. According to the transcriptome annotation results, we predicted the corresponding metabolic pathways of mannitol, cordycepin, purine nucleotides, pyrimidine nucleotides, unsaturated fatty acid, cordyceps polysaccharide and sphingolipid in $H$. sinensis for the first time, as well as the enzymes involved in each step, which can be concluded that this study provided a theoretical basis for regulation of metabolic pathways. One of the main pharmaceutically active ingredients of $H$. sinens is cordycepin [57], which is structurally similar to 2'deoxyadenosine. $H$. sinensis possesses most of the genes required for metabolism of adenine and adenosine (Additional file 4: Figure S8). For metabolic pathway of cordycepic acid in $H$. sinensis, mannitol1-phosphate phosphatase catalyzed D-mannitol-1-P to D-mannitol was not annotated in H. sinensis transcriptome, which indicated that other phosphatases took place the role of mannitol-1-phosphate phosphatase or the mannitol-1-phosphate phosphatase gene of $H$. sinensis was un-annotated to protein database since its low homology with currently reported mannitol-1-phosphate phosphatases from other organisms. In order to ensure the up- and down-regulated genes which encoding the enzymes involved in cordycepin metabolism pathway, real-time PCR was carried out to determinate differential expression genes [58]. The genes which encoding 5'-nucleotidase and $\mathrm{N}$-glycosylase were detected obviously up-regulated which consistent with the RNASeq analysis. In this present study, $5^{\prime}$-nucleotidase and $\mathrm{N}$-glycosylase were up-regulated 15.03 folds and 7.31 folds in different cultivation periods (9d-VS-3d) of $H$. sinensis, respectively. These two enzymes were significantly up-regulated when compared with other enzymes involved in cordycepin metabolic pathway. This result indicated that $5^{\prime}$-nucleotidase and $\mathrm{N}$-glycosylase played important roles in cordycepin metabolic pathway, enhancing the expression of these genes could promote biosynthesis of cordycepin. This method was helpful to ensure the gene expression levels of DEGs which could be controlled to regulate the expression level of different enzymes and achieve the desired purpose. In addition, this result will pave the theoretical foundation to carry out further research on secondary metabolic mechanisms.

As a psychrophilic fungus, $H$. sinensis could secret many low-temperature enzymes which involved in cold 
tolerance mechanism. It is found that large numbers of lipid droplets were contained in $H$. sinensis cells, as described in other fungi adapted to long-term survival in frigid conditions [59]. Compared with other fungi, H. sinensis has fewer lipases (7 vs. average 42 in other fungi) and fatty acid hydroxylases (3 vs. average 14 in other fungi), but it has a larger range and number of genes involved in triacylglycerol and fatty acid biosynthesis consistent with an emphasis on synthesizing rather than degrading lipids, and the $H$. sinensis transcriptome was enriched in fatty acid desaturases (10 vs. average 3 in other fungi) when compared with coldadapted plants and bacteria [60], indicating this fungus may respond to low temperatures by increasing membrane lipid instauration [39,45]. Considering the above possibilities, the cold tolerance mechanism of $H$. sinensis may lead to low demand of lipases and fatty acid hydroxylases, and the numbers of lipases and fatty acid hydroxylases are sufficient to $H$. sinensis adapting in frigid conditions. H. sinensis also could secrete many lowtemperature enzymes involved in the metabolism which provides the basic energy for the survival. We screened the transcriptome annotation results and found some genes of low-temperature enzymes including 2 malate dehydrogenase genes, 6 ethanol dehydrogenase genes, 5 citroyl synthetase genes and 7 chitinase genes etc., which were further obtained and verified in this study. Therefore, the analysis of transcriptomes demonstrated that $H$. sinensis has the genes encoding these low-temperature enzymes to resist to the low temperature and protect itself in the cold environment.

\section{Conclusions}

In this study, three transcriptomes of $H$. sinensis at different cultivation periods were sequenced for the first time, and the extensive transcriptomic analysis demonstrated that $H$. sinensis may have important systemic effects on $O$. sinensis at the level of genes. The genes which encoding the enzymes involved in the biosynthesis of active ingredients were predicted according to the annotation results, and the metabolic pathways of mannitol, cordycepin, purine nucleotides, pyrimidine nucleotides, unsaturated fatty acid, cordyceps polysaccharide, and sphingolipid in $H$. sinensis were described. Based on the predictions, we further investigated the interaction of different pathway networks and the corresponding enzymes. Many key or important genes involved in metabolic pathways, infection mechanism and cold tolerance mechanism were found by investigating the comparative differential expression genes from different cultivation periods. 764 DEGs between 3d and 6d, 1,869 DEGs between $3 \mathrm{~d}$ and 9d, and 770 DEGs between $6 \mathrm{~d}$ and $9 \mathrm{~d}$ were found and confirmed, respectively. These findings provide a substantial contribution and basis to further characterize the gene expression profiles for $H$. sinensis in the metabolic pathways of active ingredients.

\section{Methods}

Strains, medium and culture condition

No vertebrates or any regulated invertebrates subjects were involved in the culture and sample preparation of the $O$. sinensis and $H$. sinensis during the whole process of this study. All procedures were performed within the research guidelines of the Zhejiang University of Technology, China and did not require approval of an ethics committee.

Wild O. sinensis samples were collected from QinghaiTibet plateau in Qinghai province during May (early worm season). The wild $O$. sinensis was collected and placed in a portable refrigerator, brought back to the lab and stored at $4{ }^{\circ} \mathrm{C}$, and the isolation process of $H$. sinensis was mentioned in Additional file 4: Figure S1-S20. $H$. sinensis L0106 was isolated from the wild O. sinensis and identified according to morphology, physiology (Additional file 5: Table S4) and its 18S rRNA sequence. The H. sinensis L0106 strain was deposited at the China Center for Type Culture Collection (Wuhan, China) under accession No. CCTCC M 2011278. H. sinensis was grown on the defined medium with glucose and corn powder as carbon sources, and dried silkworm chrysalis meal and fish meal as nitrogen sources using 200-liter submerged stirred fermentors at controlled $\mathrm{pH} 7.0$ at $16^{\circ} \mathrm{C}$. Biomass samples for transcriptome analysis were taken after 3 days, 6 days and 9 days. Escherichia coli JM109 (Invitrogen, Carlsbad, CA) was used as the host for plasmid pMD18-T (Invitrogen), and Escherichia coli BL21 (DE3) (Invitrogen) was employed as a host for expression of pET28a (Invitrogen). E. coli transformants were grown in $\mathrm{LB}$ medium at $37^{\circ} \mathrm{C}$ with shaking (200 rpm).

\section{Transcriptome sequencing and analysis}

Total RNA samples from pure cultured 3 days (growth period), 6 days (pre-stable period) and 9 days (stable period) of $H$. sinensis were isolated using a standard TRIzol method and further qualified by UV determination at $260 \mathrm{~nm}$ and $280 \mathrm{~nm}$ and formaldehyde gel electrophoresis, respectively. $1 \mathrm{mg}$ of the high-quality total RNA was dissolved in $500 \mu \mathrm{l}$ of the RNase-free water, and then incubated in a water bath (Blue Pard Ltd, Shanghai, China) at $65^{\circ} \mathrm{C}$ for $10 \mathrm{~min}$. For Illumina sequencing, the total RNA extracted using TRIzol was treated with RNase-free DNase I (TaKaRa) for $30 \mathrm{~min}$ according to the manufacturer's protocols. The integrity of total RNA was checked using Agilent Technologies 2,100 Bioanalyzer (Agilent Technologies, Palo Alto, CA), and the RNA Integrity Number (RIN) value greater than eight. The mRNA was isolated from total RNA using 
Promega PolyATtract mRNA Isolation Systems [61]. The detailed procedure was carried out according to the technical manual of Promega PolyATtract mRNA Isolation Systems.

The cDNA libraries were prepared according to the manufacturer's instructions (Illumina, San Diego, CA). The poly(A) containing mRNA molecules was purified using Sera-mag Magnetic Oligo(dT) Beads (Illumina) from $20 \mathrm{mg}$ total RNA of each sample. $10 \mathrm{mM}$ Tris$\mathrm{HCl}$ was used to elute the mRNA from the magnetic beads. To avoid priming bias when synthesizing cDNA, the mRNA was first fragmented before cDNA synthesis. The mRNA was fragmented into small pieces using divalent cations at elevated temperature. The cleaned mRNA fragments primed by random primers were converted to double-stranded cDNA using SuperScript II (Invitrogen), RNase $\mathrm{H}$ (Invitrogen) and DNA Pol I (Invitrogen). The resulting cDNA was purified using the QIAquick PCR Purification Kit (Qiagen, Hilden, Germany). cDNA was then subjected to end-repair and phosphorylation using T4 DNA polymerase, Klenow DNA polymerase and T4 PNK, and subsequent purification was performed using QIAquick PCR Purification Kit (Qiagen). These repaired cDNA fragments were 30-adenylated using Klenow Exo(Illumina) and purified using MinElute PCR Purification Kit (Qiagen), producing cDNA fragments with a single 'A' base overhung at their 30-ends for subsequent adapter-ligation. Illumina PE adapters were ligated to the ends of these 30-adenylated cDNA fragments, followed by purification using MinElute PCR Purification Kit (Qiagen). To select a size range of templates for downstream enrichment, the products from ligation reactions were purified on a 2\% TAE-agarose gel (Certified Low-Range Ultra Agarose, Biorad). A range of cDNA fragments $(200 \pm 25 \mathrm{bp})$ was selected from the gel and extracted using QIAquick Gel Extraction Kit (Qiagen). Fifteen rounds of PCR amplification were performed to enrich the adapter modified cDNA library using primers complementary to the ends of the adapters. The PCR products of size $200 \pm 25$ bp were purified using QIAquick Gel Extraction Kit (Qiagen) except that Qiaquick spin columns were substituted with MinElute spin columns (Qiagen). Finally, after quantification on an Agilent Technologies 2,100 Bioanalyzer using the Agilent DNA 1,000 chip kit, the cDNA library products were sequenced using the $1 \mathrm{G}$ Illumina Genome Analyzer. Two biological replicates of every sample were analyzed.

After checking and filtering steps, the SOAPdenovo software (http://soap.genomics.org.cn/soapdenovo.html) was used to carry out the whole transcriptome assembly [62]. The contigs without any gap were obtained after SOAPdenovo assembly and correction. Subsequently, the obtained reads were aligned onto the contigs sequences and aligned paired ends. Meanwhile, the relationship and rate of consistent between each pair of contigs were evaluated, and then the scaffolds were constructed step by step. Paired-end reads were used again for gap filling of scaffolds to get sequences with least Ns and cannot be extended on either end. Such sequences were defined as unigenes.

\section{Unigene function annotation}

Unigene annotation provides information of expression and functional annotation of unigene. Information of functional annotation includes protein functional annotation, COG functional annotation and Gene Ontology (GO) (http://geneontology.org/) functional annotation of unigenes. Unigene sequences are firstly aligned by blastx to protein databases like $\mathrm{nr}$ (http://www.ncbi.nlm.nih. gov/sites/entrez?db=protein), Swiss-Prot (http://www. expasy.org), KEGG (http://www.kegg.jp/) and COG (E-value < 0.00001) (http://www.ncbi.nlm.nih.gov/COG/), retrieving proteins with the highest sequence similarity with the given unigenes along with their protein functional annotations. KEGG database contains systematic analysis of inner-cell metabolic pathways and functions of gene products, which helps to study the complicated biological behaviors of genes. Pathway annotation of unigenes was obtained by KEGG database, and then the unigenes are aligned to COG database to predict and classify possible functions.

\section{Gene ontology analysis}

The gene ontology terms of each $H$. sinensis gene were obtained by the software Blast2GO (http://www.blast2go. com) using the default parameters. Blast2GO was also used for GO functional enrichment analysis of certain genes, by performing Fisher's exact test with robust false discovery rate (FDR) correction to obtain an adjusted $p$-value between certain test gene groups and the whole annotation.

\section{Pathway analysis with KEGG}

Pathway analysis was carried out according to KEGG mapping method [63]. Enzyme commission (EC) numbers were assigned to unique sequences that had BLASTX scores with cutoff values of $\mathrm{E}<1.0 \mathrm{e} 5$, as determined based on searching the protein databases. The sequences were mapped to the KEGG biochemical pathways according to the EC distribution in the pathway database.

\section{Normalized expression level of gene by RNA-Seq}

The expression level of gene by RNA-Seq was normalized by the number of reads per kilobase of exon region per million mapped reads (RPKM) [64]. The cutoff value for determining gene transcriptional activity was determined based on a 95\% confidence interval for all RPKM values of each gene. 


\section{Discovery of novel transcripts}

Novel transcripts can be found by high throughput sequencing [24] since present databases may be incomplete. Gene models found in intergenic regions (200 bp away from upstream or downstream genes) were thought to be candidate of novel transcripts.

\section{Refinement of gene structures}

The gene structure was optimized according to the distribution of the reads, information of paired-end and the annotation of reference gene. The distribution of reads in the transcriptome was obtained by aligning the continuous and overlap reads form a Transcription Active Region (TAR). According to paired-end data, different TARs were connected to form a potential gene model. Subsequently, the gene model with the existing gene annotated to extend the gene $5^{\prime}$ and $3^{\prime}$ end was further compared.

\section{Alternative splicing analysis}

Alternative splicing analysis [65] was developed in this study. There are mainly four types of alternative splicing in the $H$. sinensis transcriptome analysis including ES, IR, A5SS and A3SS. The following algorithms were used to detect alternative splicing events. First, junction sites giving information about boundaries and combinations of different exons in a transcript, are detected by TopHat (with all default parameters) [66]. All junction sites of the same gene are used to distinguish type of its alternative splicing event. If junctions were detected, it indicated that exon skipping occurred in transcript. If the junctions meet the following conditions, there is an Intron Retention event between Exon1 and Exon2. 1) Junction 1 is detected, which means there is an intron between Exon1 and Exon2; 2) 90\% of this intron is covered by unique-mapping reads; 3 ) the coverage depth of the intron is at least 15\% of the coverage depth of Exon1 or Exon2; 4) 5 bp both upstream and downstream of this intron's both boundaries is required to be covered by reads; 5) the intron region cannot be covered by other genes. If either Junction 2 or Junction 3, which has the same $3^{\prime}$ but different $5^{\prime}$ splice sites with Junction 1 , is detected, then there is an Alternative $5^{\prime}$ Splice Site event between Exon1 and Exon2. If either Junction 2 or Junction 3, which has the same $5^{\prime}$ but different $3^{\prime}$ splice sites with Junction 1, is detected, then there is an Alternative 3' Splice Site event between Exon1 and Exon2.

\section{Verification of predicted enzymes in metabolic pathways} In this study, we used bioinformatics softwares including DNAMAN (Version 5.2.2, Lynnon Biosoft, Canada) and Primer 5.0 (http://www.primer-e.com), and online bioinformatic tools such as KEGG pathway database (http://www. kegg.jp/), Blast (http://blast.ncbi.nlm.nih.gov/Blast.cgi) and
ORF Finder (http://www.ncbi.nlm.nih.gov/gorf/gorf.html) to analyze metabolic pathways of seven main bioactive ingredients including mannitol, cordycepin, purine nucleotides, pyrimidine nucleotides, unsaturated fatty acid, cordyceps polysaccharide and sphingolipid in $H$. sinensis. The functional genes and enzymes which were involved in metabolic pathways of $H$. sinensis were then verified by using gene cloning and protein expression according to the previously reported methods [67].

PCR was performed to validate the genes which can encode the enzymes involved in different pathways. Primers were designed according to the predicted ORF sequences of target genes (Additional file 6: Table S5-S7). Genes related to synthesis pathways were cloned by PCR amplification method. Subsequently, the genes were sub-cloned to the pMD18-T vector (Invitrogen). Finally, the recombinant plasmid was successfully transformed to E. coli JM109 competent cells (Invitrogen). The recombinant E. coli JM109 was cultivated in the shaker at $200 \mathrm{rpm}$ and then the recombinant plasmids were extracted using plasmid extraction kit (Qiagen). After sequencing, the target genes were sub-cloned into the pET28a (Invitrogen), the recombinant plasmid was also successfully transferred to E. coli BL21 competent cells (Invitrogen). Then the genes were expressed in E. coli BL21 using IPTG as inducer. The expressed proteins were further detected by SDSPAGE [68].

\section{Sequence submission}

The 18S rRNA sequence of $H$. sinensis has been deposited in a public repository of NCBI GenBank Database (http://www.ncbi.nlm.nih.gov/WebSub/?tool=genbank) with a GenBank accession number of KP090933. The gene and protein sequences of hexokinase (HK-1), hexokinase (HK-3), hexokinase (HK-4), hexokinase (HK-5), hexokinase (HK-6), hexokinase (HK-8), glucosephosphate isomerase (GPI-1), glucosephosphate isomerase (GPI-2), glucosephosphate isomerase (GPI-3), mannitol1-phosphate 5-dehydrogenase (mtlD-1), mannitol-1phosphate 5-dehydrogenase (mtlD-2), ADP-ribose pyrophosphatase (ADPR-PPase), ribose-phosphate pyrophosphokinase (PRPS-1), ribose-phosphate pyrophosphokinase (PRPS-2), amidophosphoribosyltransferase (purF-2), amidophosphoribosyltransferase (purF-3), phosphoribosylamine-glycine ligase (purD-1), phosphoribosylglycinamide formyltransferase (GAR TFase-1), phosphoribosylformylglycinamidine synthase (purL-1), phosphoribosylformylglycinamidine cyclo-ligase (purM-1), phosphoribosylaminoimidazole carboxylase (PAICS-1), phosphoribosylaminoimidazole-succinocarboxamide synthase (purC-1), adenylosuccinate lyase (purB-1), phosphoribosylaminoimidazolecarboxamide formyltransferase (purH-1), adenylosuccinate synthase (purA-2), 
$5^{\prime}$-nucleotidase (5' -Nuc-1), $5^{\prime}$-nucleotidase ( $5^{\prime}$-Nuc-2), $\mathrm{N}$ glycosylase/DNA lyase (N-Gly-1), purine nucleosidase (iunH-1), adenosine kinase (ADK-1), adenine phosphoribosyltransferase (APRT-1), AMP deaminase (AMPD-1), AMP deaminase (AMPD-6), IMP dehydrogenase (guaB-1), IMP dehydrogenase (guaB-2), GMP synthase (guaA-1), GMP synthase (guaA-2), GMP synthase (guaA-3), guanine deaminase (guaD-1), guanine deaminase (guaD-2) and xanthine dehydrogenase/oxidase (XDH-1), which were verified by experiments in this study, have been deposited in the NCBI GenBank Database, and the GenBank accession numbers for these sequences were recorded as KP090934, КР090935, КР090936, КР090937, КР090938, КР090939, КР090940, КР090941, КР090942, КР090943, КР090944, КР090945, КР090946, КР090947, КР090948, КР090949, КР090950, КР090951, КР090952, КР090950, КР090953, КР090954, КР090955, КР090956, КР090957, КР090958, КР090959, КР090960, КР090961, КР090962, КР090963, КР090964, КР090965, КР090966, КР090967, КР090968, КР090969, КР090970, КР090971, КР090972 and KP090973, respectively. The detailed gene and protein sequences were shown in Additional file 7.

\section{Additional files}

Additional file 1: Table S1. Unigene annotations provide functional annotations of unigene (All) and expression levels. Functional annotations of unigene including protein sequence similarity, KEGG Pathway, COG and Gene Ontology (GO).

Additional file 2: Table S2. COG function classification of $H$. sinensis unigenes (All) compared with $O$. sinensis grass-part (OSGP) and $O$. sinensis worm-part (OSWP)

Additional file 3: Table S3. Statistics of $H$. sinensis transcriptome mapped to reference genome and reference gene.

Additional file 4: Figures S1-S20. Supplementary results and methods, figure legends for supplementary figures and tables.

Additional file 5: Table S4. Biolog metabolic fingerprinting analysis of H. sinensis.

Additional file 6: Table S5. The primers used for cloning and expressing genes involved in mannitol metabolic pathway. Table S6. The primers used for cloning and expressing genes involved in cordycepin metabolic pathway. Table S7. The primers used for cloning and expressing genes involved in purine nucleotides metabolic pathway. Table S8. The primers used for real-time PCR involved in mannitol metabolic pathway. Table S9. The primers used for real-time PCR involved in cordycepin metabolic pathway. Table S10. The primers used for real-time PCR involved in purine nucleotides metabolic pathway.

Additional file 7: List of $18 \mathrm{~S}$ rRNA gene, mannitol anabolic functional genes, cordycepin anabolic functional genes and purine nucleotides anabolic functional genes including GenBank accession numbers.

\section{Competing interests}

The authors declare that they have no competing interests.

\section{Authors' contributions}

ZQL, SL PJB and HYW conceived the study and participated in its design and coordination. ZQL, SL HW, FX and YQZ participated in the design of the study. ZQL oversaw the transcriptome assembly, automatic transcriptome annotations, and conducted the transcriptome comparisons. SL and LFW conducted in the transcriptome assembly and automatic transcriptome annotations. MEB and XRW carried out the bioinformatic analyses, designed and implemented the database. ZQL SL, PJB and YGZ drafted the manuscript. All authors read and approved the final manuscript.

\section{Acknowledgements}

We would like to thank the anonymous reviewers for the helpful comments. This work was financially supported by the National High Technology Research and Development Program of China (no. 2012AA021701) and the Key Scientific and Technology Programs of Zhejiang Province (no. 2012C03005-1).

\section{Author details}

Institute of Bioengineering, Zhejiang University of Technology, Hangzhou 310014, Zhejiang, P R China. 'East China Pharmaceutical Group Limited Co., Ltd, Hangzhou 311000, Zhejiang, P R China. ${ }^{3}$ Polytechnic School of Engineering, New York University, 6 MetroTech Center, Brooklyn, NY 11201, USA.

Received: 10 July 2014 Accepted: 22 January 2015

Published online: 21 February 2015

\section{References}

1. Jiang Y, Yao YJ. Names related to Cordyceps sinensis anamorph. Mycotaxon. 2002;84:245-54.

2. Dong $\mathrm{CH}$, Yao YJ. Nutritional requirements of mycelial growth of Cordyceps sinensis in submerged culture. J Appl Microbiol. 2005;99(3):483-92

3. Zhang Y, Li E, Wang C, Li Y, Liu X. Ophiocordyceps sinensis, the flagship fungus of China: terminology, life strategy and ecology. Mycology. 2012:3(1):2-10.

4. Winkler D. Yartsa Gunbu (Cordyceps sinensis) and the fungal commodification of Tibet's rural economy. Econ Bot. 2008:62(3):291-305.

5. Zhou X, Gong Z, Su Y, Lin J, Tang K. Cordyceps fungi: natural products, pharmacological functions and developmental products. J Pharm Pharmcol. 2009;61(3):279-91

6. Sharma S. Trade of Cordyceps sinensis from high altitudes of the Indian Himalaya: conservation and biotechnological priorities. Curr Sci. 2004;86(12):1614-9.

7. Li C, Li Z, Fan M, Cheng W, Long Y, Ding T, et al. The composition of Hirsutella sinensis, anamorph of Cordyceps sinensis. J Food Compos Anal. 2006;19(8):800-5

8. Koh JH, Yu KW, Suh HJ, Choi YM, Ahn TS. Activation of macrophages and the intestinal immune system by an orally administered decoction from cultured mycelia of Cordyceps sinensis. Biosci Biotechnol Biochem. 2002:66(2):407-11

9. Cheung JK, Li J, Cheung AW, Zhu Y, Zheng KY, Bi CW, et al. Cordysinocan, a polysaccharide isolated from cultured Cordyceps, activates immune responses in cultured T-lymphocytes and macrophages: Signaling cascade and induction of cytokines. J Ethnopharmacol. 2009;124(1):61-8.

10. Zhang Y, Liu X, Wang M. Cloning, expression, and characterization of two novel cuticle-degrading serine proteases from the entomopathogenic fungus Cordyceps sinensis. Res Microbiol. 2008;159(6):462-9.

11. Buenz E, Bauer B, Osmundson T, Motley T. The traditional Chinese medicine Cordyceps sinensis and its effects on apoptotic homeostasis. J Ethnopharmacol. 2005;96(1):19-29.

12. Chen $Y Q$, Wang N, Qu LH, Li TH, Zhang WM. Determination of the anamorph of Cordyceps sinensis inferred from the analysis of the ribosomal DNA internal transcribed spacers and 5.85 rDNA. Biochem Syst Ecol. 2001;29(6):597-607

13. Zhao J, Wang N, Chen Y, Li T, Qu L. Molecular identification for the asexual stage of Cordyceps sinensis. Acta Scientiarum Naturalium Universitatis Sunyatseni. 1998;38(1):121-3.

14. Yue GGL, Lau CBS, Fung KP, Leung PC, Ko WH. Effects of Cordyceps sinensis, Cordyceps militaris and their isolated compounds on ion transport in Calu-3 human airway epithelial cells. J Ethnopharmacol. 2008;117(1):92-101.

15. Velculescu VE, Zhang L, Vogelstein B, Kinzler KW. Serial analysis of gene expression. Science. 1995;270(5235):484-6.

16. Bishop J, Morton J, Rosbash M, Richardson M. Three abundance classes in HeLa cell messenger RNA. Nature. 1974;250(463):199-204. 
17. Schena M, Shalon D, Davis RW, Brown PO. Quantitative monitoring of gene expression patterns with a complementary DNA microarray. Science. 1995;270(5235):467-70

18. Kawai J, Shinagawa A, Shibata K, Yoshino M, Itoh M, Ishii Y, et al. Functional annotation of a full-length mouse cDNA collection. Nature. 2001;409(6821):685-90.

19. Mortazavi A, Williams BA, Mccue K, Schaeffer L, Wold B. Mapping and quantifying mammalian transcriptomes by RNA-Seq. Nat Methdos. 2008:5(7):621-8

20. Wang ET, Sandberg R, Luo S, Khrebtukova I, Zhang L, Mayr C, et al. Alternative isoform regulation in human tissue transcriptomes. Nature. 2008;456(7221):470-6.

21. Schunter C, Vollmer SV, Macpherson E, Pascual M. Transcriptome analyses and differential gene expression in a non-model fish species with alternative mating tactics. BMC Genomics. 2014;15:167.

22. Ma L, Ma Q, Li X, Cheng L, Li K, Li S. Transcriptomic analysis of differentially expressed genes in the Ras1(CA)-overexpressed and wildtype posterior silk glands. BMC Genomics. 2014;15:182

23. Fu X, Fu N, Guo S, Yan Z, Xu Y, Hu H, et al. Estimating accuracy of RNA-Seq and microarrays with proteomics. BMC Genomics. 2009;10:161.

24. Wang Z, Gerstein M, Snyder M. RNA-Seq: a revolutionary tool for transcriptomics. Nat Rev Genet. 2009;10(1):57-63.

25. Vera JC, Wheat CW, Fescemyer HW, Frilander MJ, Crawford DL, Hanski I, et al. Rapid transcriptome characterization for a nonmodel organism using 454 pyrosequencing. Mol Ecol. 2008;17(7):1636-47.

26. Maussion G, Yang J, Suderman M, Nagy C, Arnovitz M, Mechawar N, et al. Functional DNA methylation in a transcript specific 3'UTR region of TrkB associates with suicide. Epigenetics. 2014;9(8):1061-70.

27. Zhang G, Guo G, Hu X, Zhang Y, Li Q, Li R, et al. Deep RNA sequencing at single base-pair resolution reveals high complexity of the rice transcriptome. Genome Res. 2010;20(5):646-54

28. Zhang $\mathrm{S}$, Feng $\mathrm{H}$, Li X, Jin Y, Dong W. Genome research profile of two Cordyceps sinensis cDNA libraries. Chinese Sci Bull. 2010;55(14):1403-11.

29. Black DL. Mechanisms of alternative pre-messenger RNA splicing. Annu Rev Biochem. 2003;72(1):291-336.

30. Stamm S, Ben Ari S, Rafalska I, Tang Y, Zhang Z, Toiber D, et al. Function of alternative splicing. Gene. 2005;344:1-20.

31. Lareau LF, Green RE, Bhatnagar RS, Brenner SE. The evolving roles of alternative splicing. Curr Opin Struc Biol. 2004;14(3):273-82.

32. Pan $Q$, Shai $O$, Lee $L J$, Frey BJ, Blencowe BJ. Deep surveying of alternative splicing complexity in the human transcriptome by high-throughput sequencing. Nat Genet. 2008;40(12):1413-5.

33. Sultan M, Schulz MH, Richard $H$, Magen A, Klingenhoff A, Scherf M, et al. A global view of gene activity and alternative splicing by deep sequencing of the human transcriptome. Science. 2008:321(5891):956-60.

34. Tang F, Barbacioru C, Wang Y, Nordman E, Lee C, Xu N, et al. mRNA-Seq whole-transcriptome analysis of a single cell. Nat Methods. 2009;6(5):377-82.

35. Ner Gaon H, Halachmi R, Savaldi Goldstein S, Rubin E, Ophir R, Fluhr R. Intron retention is a major phenomenon in alternative splicing in Arabidopsis. Plant J. 2004;39(6):877-85

36. Ho EC, Cahill MJ, Saville BJ. Gene discovery and transcript analyses in the corn smut pathogen Ustilago maydis: expressed sequence tag and genome sequence comparison. BMC Genomics. 2007:8(1):334.

37. Ebbole DJ, Jin Y, Thon M, Pan H, Bhattarai E, Thomas T, et al. Gene discovery and gene expression in the rice blast fungus, Magnaporthe grisea: analysis of expressed sequence tags. Mol Plant Microbe In. 2004:17(12):1337-47.

38. Wu YL, Sun CR, Pan YJ. Studies on isolation and structural features of a polysaccharide from the mycelium of an Chinese edible fungus (Cordyceps sinensis). Carbohyd Polym. 2006;63(2):251-6.

39. Hu X, Zhang YJ, Xiao GH, Zheng P, Xia YL, Zhang XY, et al. Genome survey uncovers the secrets of sex and lifestyle in caterpillar fungus. Chinese Sc Bull. 2013;58(23):2846-54

40. Zheng P, Xia Y, Xiao G, Xiong C, Hu X, Zhang S, et al. Genome sequence of the insect pathogenic fungus Cordyceps militaris, a valued traditional Chinese medicine. Genome Biol. 2011;12(11):R116.

41. Morozova O, Marra MA. Applications of next-generation sequencing technologies in functional genomics. Genomics. 2008:92(5):255-64.

42. van den Berg MA, Albang R, Albermann K, Badger JH, Daran JM, Driessen $\mathrm{AJ}$, et al. Genome sequencing and analysis of the filamentous fungus Penicillium chrysogenum. Nat Biotechnol. 2008;26(10):1161-8.
43. Pel HJ, De Winde JH, Archer DB, Dyer PS, Hofmann G, Schaap PJ, et al. Genome sequencing and analysis of the versatile cell factory Aspergillus niger CBS 513.88. Nat Biotechnol. 2007;25(2):221-31.

44. Gao Q, Jin K, Ying SH, Zhang Y, Xiao G, Shang Y, et al. Genome sequencing and comparative transcriptomics of the model entomopathogenic fungi Metarhizium anisopliae and M. acridum. PLoS Genet. 2011;7(1):e1001264.

45. Stone R. Mycology. Last stand for the body snatcher of the Himalayas? Science. 2008;322(5905):1182.

46. Clarkson JP, Staveley J, Phelps K, Young CS, Whipps JM. Ascospore release and survival in Sclerotinia sclerotiorum. Mycol Res. 2003;107(2):213-22.

47. Zacharuk R. Fine structure of the fungus Metarrhizium anisopliae infecting three species of larval Elateridae (Coleoptera). IV. Development within the host. Can J Microbiol. 1971:17(4):525-9.

48. Zacharuk R. Fine structure of the fungus Metarrhizium anisopliae infecting three species of larval Elateridae (Coleoptera): III. Penetration of the host integument. J Invertebr Pathol. 1970;15(3):372-96.

49. Clarkson JM, Charnley AK. New insights into the mechanisms of fungal pathogenesis in insects. Trends Microbiol. 1996;4(5):197-203.

50. Samuels R, Paterson I. Cuticle degrading proteases from insect moulting fluid and culture filtrates of entomopathogenic fungi. Biochem Mol Biol. 1995:110(4):661-9.

51. St L, Joshi L, Bidochka MJ, Rizzo NW, Roberts DW. Characterization and ultrastructural localization of chitinases from Metarhizium anisopliae, M. flavoviride, and Beauveria bassiana during fungal invasion of host (Manduca sexta) cuticle. Appl Environ Microbiol. 1996;62(3):907-12.

52. Charnley AK, StLeger RJ. The role of cuticle-degrading enzymes in fungal pathogenesis in insect. In: Cole GT, Hoch HC, editors. The fungal spore and disease initiation in plants and animals. US: Springer; 1991. p. 267-86.

53. Wang ZB, Li N, Wang M, Wang Y, Du L, Ji XF, et al. Simultaneous determination of nucleosides and their bases in Cordyceps sinensis and its substitutes by matrix solid-phase dispersion extraction and HPLC. J Sep Sci. 2013;36(14):2348-57.

54. Chen PX, Wang SA, Nie SP, Marcone M. Properties of Cordyceps sinensis: A review. J Funct Foods. 2013;5(2):550-69.

55. Nie S, Cui SW, Xie M, Phillips AO, Phillips GO. Bioactive polysaccharides from Cordyceps sinensis: Isolation, structure features and bioactivities. Bioact Carb Diety Fibr. 2013;1(1):38-52.

56. Lennon MB, Suhadolnik RJ. Biosynthesis of 3'-deoxyadenosine by Cordyceps militaris: Mechanism of reduction. Nucleic Acids Protein Synth. 1976;425 (4):532-6.

57. Zuo HL, Chen SJ, Zhang DL, Zhao J, Yang FQ, Xia ZN. Quality evaluation of natural Cordyceps sinensis from different collecting places in China by the contents of nucleosides and heavy metals. Anal Methods. 2013:5(20):5450-6.

58. Livak KJ, Schmittgen TD. Analysis of relative gene expression data using real-time quantitative PCR and the $2^{-\Delta \Delta C t}$ method. Methods. 2001;25(4):402-8.

59. Amaretti A, Raimondi S, Sala M, Roncaglia L, De Lucia M, Leonardi A, et al. Single cell oils of the cold-adapted oleaginous yeast Rhodotorula glacialis DBVPG 4785. Microb Cell Fact. 2010;9(1):73.

60. D'Amico S, Collins T, Marx JC, Feller G, Gerday C. Psychrophilic microorganisms: challenges for life. EMBO Rep. 2006;7(4):385-9.

61. Burke P. Technically speaking: PolyATtract ${ }^{\circledR}$ mRNA Isolation Systems. Promega Notes. 1996;56:27-9.

62. Wang Z, Fang B, Chen J, Zhang X, Luo Z, Huang L, et al. De novo assembly and characterization of root transcriptome using Illumina paired-end sequencing and development of CSSR markers in sweetpotato (Ipomoea batatas). BMC Genomics. 2010;11(1):726

63. Kanehisa M, Araki M, Goto S, Hattori M, Hirakawa M, Itoh M, et al. KEGG for linking genomes to life and the environment. Nucleic Acids Res. 2008;36 suppl 1:D480-4

64. Wagner GP, Kin K, Lynch VJ. Measurement of mRNA abundance using RNA-Seq data: RPKM measure is inconsistent among samples. Theor Biosci. 2012;131(4):281-5.

65. Blencowe BJ. Alternative splicing: New insights from global analyses. Cell. 2006;126(1):37-47.

66. Trapnell C, Pachter L, Salzberg SL. TopHat: discovering splice junctions with RNA-Seq. Bioinformatics. 2009;25(9):1105-11. 
67. Reboul J, Vaglio P, Rual JF, Lamesch P, Martinez M, Armstrong CM, et al. C-elegans ORFeome version 1.1: experimental verification of the genome annotation and resource for proteome-scale protein expression. Nat Genet. 2003;34(1):35-41.

68. Chong L. Molecular cloning: A laboratory manual, 3rd edition. Science. 2001;292(5516):446.

Submit your next manuscript to BioMed Central and take full advantage of:

- Convenient online submission

- Thorough peer review

- No space constraints or color figure charges

- Immediate publication on acceptance

- Inclusion in PubMed, CAS, Scopus and Google Scholar

- Research which is freely available for redistribution 